\title{
Desempenho de leitura e escrita em usuários de implante coclear: revisão integrativa
}

\author{
Reading and writing performance in cochlear implant users: \\ integrative review
}

\author{
Kryssia Layane Santos de Oliveira' ${ }^{1}$, Antonio Lucas Ferreira Feitosa ${ }^{1}$ (D), Gabriel Trevizani Depolli ${ }^{2}$ (D), \\ Cristiane Monteiro Pedruzzi' ${ }^{1}$
}

\section{RESUMO}

Objetivos: Descrever o desempenho de leitura e da escrita em usuários de implante coclear por meio de uma revisão integrativa. Estratégia de pesquisa: A busca dos estudos ocorreu nas plataformas: SciELO, PubMed e Biblioteca Virtual em Saúde as quais abrangem as bases de dados: MEDLINE e LILACS. A questão norteadora desta revisão foi: como se apresenta o desempenho de leitura e da escrita em usuários de implante coclear? Critérios de seleção: Estudos publicados nos últimos cinco anos, disponíveis nos idiomas português e inglês, que descreveram o desempenho de leitura e escrita em usuários de implante coclear. Dois autores revisaram e extraíram os dados como: ano, tipo de pesquisa, país, amostra, objetivo, idade de implante coclear e conclusões. Resultados: Foram inclusos oito artigos. A faixa etária variou entre cinco e 18 anos de idade. Países como Espanha e Irã publicaram com mais frequência estudos nesta área. No total, foram avaliados 419 escolares, sendo que 238 eram usuários de implante coclear, que tinham como grupo controle 181 crianças com audição normal. Estudos demonstram que usuários com implante coclear possuem atrasos significativos durante o processo de desenvolvimento de leitura e de escrita, considerado ainda maior quando comparados com indivíduos de audição normal. Conclusão: Mesmo com o uso do implante coclear, o desempenho de leitura e de escrita em escolares pode ser considerado pior em comparação ao de indivíduos com audição normal. Além disso, o desempenho de leitura e de escrita dos participantes está aquém do esperado para faixa etária e ano escolar.

Palavras-chave: Criança; Educação; Implante coclear; Leitura; Perda auditiva

\begin{abstract}
Purpose: To describe the reading and writing performance of cochlear implant users, through an integrative review. Research strategy: The search for studies took place on the platforms: SciELO, PubMed and Virtual Health Library, which includes the databases: MEDLINE and LILACS. The guiding question of this review was: how is the reading and writing performance of cochlear implant users? Selection criteria: Studies published in the last five years, available in Portuguese and English, which described the reading and writing performance of cochlear implant users. Two authors reviewed and selected data such as: year, type of research, country, sample, objective, age of cochlear implant and conclusions. Results: Eight articles were included. The age range varied between 5 and 18 years of age. Spain and Iran have published studies in this area more frequently. In total, 419 schoolchildren were evaluated, 238 of whom were cochlear implant users, with a control group of 181 children with normal hearing. Studies demonstrate cochlear implant users have delays during reading and writing development, even more when compared to people with normal hearing. Conclusion: Even with the use of the cochlear implant, reading and writing performance in schoolchildren is considerably worse compared to individuals with normal hearing. In addition, the reading and writing performance of the participants is below expectations for the age group and school year.
\end{abstract}

Keywords: Child; Education; Cochlear implantation; Reading; Hearing loss

\footnotetext{
Trabalho realizado na Universidade Estadual de Ciências da Saúde de Alagoas - UNCISAL - Maceió (AL), Brasil.

${ }^{1}$ Universidade Estadual de Ciências da Saúde de Alagoas - UNCISAL - Maceió (AL), Brasil.

${ }^{2}$ Universidade Federal do Espírito Santo - UFES - Vitória (ES), Brasil.

Conflito de interesses: Não.

Contribuição dos autores: KLSO participou da idealização do estudo, coleta, análise e interpretação dos dados e redação do artigo; ALFF e GTD participaram da coleta, análise e interpretação dos dados; CMP participou, na condição de orientadora, da idealização do estudo, análise, interpretação dos dados e redação do artigo. Financiamento: Nada a declarar.

Autor correspondente: Cristiane Monteiro Pedruzzi. E-mail: cristiane-pedruzzi@ig.com.br

Recebido: Janeiro 30, 2020; Aceito: Maio 19, 2020
} 


\section{INTRODUÇÃO}

O implante coclear (IC) é um dispositivo biomédico, cirurgicamente implantado na cóclea em pacientes com perda auditiva severa/profunda que não apresentaram resultados satisfatórios com o uso do Aparelho de Amplificação Sonora Individual (AASI). Esse dispositivo tem como objetivo desempenhar as funções das células ciliadas internas da cóclea, estimulando eletricamente as fibras remanescentes do nervo auditivo $^{(1)}$. Os primeiros anos de vida são fundamentais para a realização da cirurgia do IC. Neste período, acontece o auge do processo de maturação do sistema auditivo central e da plasticidade neuronal da via auditiva, etapa fundamental para o desenvolvimento das habilidades auditivas e de linguagem ${ }^{(2)}$.

Por outro lado, é difícil prever como os indivíduos pré-linguais com uso do IC nos três primeiros anos de vida organizam as informações auditivas recebidas e até quando desenvolvem linguagem ${ }^{(3)}$. Isso porque o progresso auditivo depende de vários fatores, como a qualidade da audição pré-implante, a causa da perda auditiva, o tempo de privação sensorial, a idade em que o usuário recebeu o IC, o tipo de abordagem da reabilitação auditiva e de linguagem do usuário e a própria capacidade de neuroplasticidade do organismo(4).

Com isso, o input auditivo incompleto antes do uso do IC pode afetar o desenvolvimento da linguagem do indivíduo, incluindo os campos semântico/lexical, gramatical/sintático, níveis fonológicos e pragmáticos. $\mathrm{O}$ nível de discriminação e compreensão auditiva também pode sofrer influências, conforme as inúmeras variáveis que interferem nos benefícios do uso do $\mathrm{IC}^{(5)}$.

Desde 1957, quando foi relatada a primeira experiência de estimulação do nervo auditivo por meio da introdução de um eletrodo no ouvido de uma pessoa com perda auditiva profunda ${ }^{(6)}$, o número de crianças que recebem o IC aumenta gradativamente $^{(7)} \mathrm{e}$, consequentemente, as escolas têm recebido com mais frequência estudantes com o uso de $\mathrm{IC}^{(8)}$. As condições de aprendizagem dos conteúdos escolares para alunos com uso de IC têm se mostrado deficitárias ${ }^{(9,10)}$.

Embora a tecnologia do IC propicie experiências auditivas que aprimoram o desenvolvimento da linguagem oral, seus usuários enfrentam dificuldades e desafios semelhantes, advindas de um atraso na aquisição da linguagem oral e escrita ${ }^{(11)}$.

Estudos relatam que usuários de IC possuem um menor desempenho de leitura e de escrita, quando comparados a indivíduos com audição normal ${ }^{(12)}$. Esta dificuldade pode ser resultado do seu déficit sensorial, mas também por outros fatores como o ambiente, que as tornam crianças de risco para aquisição gradual do desenvolvimento da linguagem e, consequentemente, na aprendizagem do conteúdo escolar em geral ${ }^{(13)}$.

O desempenho na leitura e na escrita de usuários de IC é muito estudado em países do primeiro mundo ${ }^{(13)}$. Entretanto, na literatura brasileira, este assunto ainda é pouco discutido, inclusive os protocolos de avaliação de desempenho de leitura e escrita brasileiros são padronizados para as crianças ouvintes ${ }^{(14)}$.

Considerando-se a hipótese de que o desenvolvimento da leitura e da escrita em usuários de IC oralizados pode ocorrer de maneira semelhante ao do ouvinte com hipóteses auditivas e fonéticas, o objetivo deste estudo foi descrever o desempenho de leitura e de escrita em usuários de IC, por meio de uma revisão integrativa.

\section{ESTRATÉGIA DE BUSCA}

Trata-se de um estudo do tipo revisão integrativa, que selecionou, reuniu, analisou e discutiu achados desenvolvidos a partir de diferentes metodologias, permitindo aos revisores sintetizá-los sem ferir a filiação epistemológica dos estudos empíricos incluídos ${ }^{(15)}$.

Para operacionalizar a revisão, foram seguidas seis etapas: identificação do tema e seleção da hipótese; busca dos estudos nas bases de dados; avaliação dos estudos; extração de dados dos estudos; análise dos resultados e apresentação da revisão, tendo em vista a questão norteadora: Como se apresenta o desempenho de leitura e da escrita em usuários de IC?

A busca dos estudos ocorreu nas principais plataformas de dados: Scientific Electronic Library Online (SciELO), a maior base de dados brasileira; PubMed e Biblioteca Virtual em Saúde (BVS) que abrange as bases de dados: Medical Literature Analysis and Retrieval System Online (MEDLINE), maior base de dados médica americana e Literatura Latino-Americana e do Caribe em Ciências da Saúde (LILACS), maior bases de dados latino-americana. A busca foi realizada durante os meses de agosto e setembro de 2019.

Quatro descritores, em português e inglês, controlados pelos Descritores em Ciências da Saúde (DeCS) foram utilizados: implante coclear, educação, criança e leitura, associados às palavras-chave: escrita, aparelho auditivo e implante auditivo, utilizando o operador booleano " $A N D$ " entre os descritores $\mathrm{e}$ "OR" entre as palavras-chave, a fim de ampliar a busca.

Dez combinações de estratégias de busca foram feitas utilizando os descritores e palavras-chave em português, assim como em inglês. A seguir, exemplifica-se a estratégia de busca em inglês, a saber: "Cochlear Implantation" AND Child AND Writing; "Cochlear Implantation" OR "Cochlear Implantations" AND Child AND Writing; "Cochlear Implantation" OR "Implantation Auditory" AND Child AND Education; "Cochlear Implantation" OR "Implantation Auditory" AND Child AND Reading AND "Cochlear Implantation" OR "Implantation Auditory" AND Child AND Writing; "Cochlear Implantation" OR "Implantation Auditory" AND Reading AND "Cochlear Implantation" OR "Implantation Auditory" AND writing; "Cochlear Implantation" OR "Implantation Auditory" OR "Cochlear Implantation" $A N D$ Child AND education; "Cochlear Implantation" OR "Implantation Auditory" OR "Cochlear Implantations" AND child AND Reading; "Cochlear Implantation" OR "Implantation Auditory" OR "Cochlear Implantations" AND Reading; "Cochlear Implantation" OR "Implantation Auditory" OR "Cochlear Implantations" AND Writing;

\section{CRITÉRIOS DE SELEÇÃo}

Os critérios de inclusão estabelecidos para esta revisão englobam estudos publicados nos últimos cinco anos (2014-2019), disponíveis em qualquer idioma, que descreveram o desempenho de leitura e escrita de crianças usuárias de IC inseridas em programa de reabilitação aurioral.

Como critérios de exclusão, foi estabelecido que não seriam inclusos: artigos de revisões (sistemática, narrativa ou integrativa), relatos de casos, teses, dissertações, artigos de opinião, resenhas, estudos que tinham como amostra indivíduos com comorbidades além da perda auditiva, ou repetidos pela 
sobreposição de bases de dados, ou aqueles que não descreveram o desempenho de leitura e escrita de usuários de IC.

Os artigos foram selecionados de forma autônoma por dois avaliadores, que leram de forma independente todos os títulos e resumos dos estudos obtidos por meio das estratégias de busca pré-definidas. Nos casos que ocorreram discordância da inclusão de algum artigo, um terceiro avaliador foi solicitado a arbitrar. Com isso, para a seleção final dos artigos, foi realizada a leitura integral dos artigos selecionados, bem como a busca das informações necessárias sobre o desempenho de leitura e escrita em usuários de IC.

\section{Análise dos dados}

Após a seleção dos artigos, foi realizada leitura na íntegra destes estudos. Para melhor apresentação dos dados encontrados, foi preciso considerar as seguintes variáveis: autor e ano, tipo de estudo, revista, fator de impacto, país, amostra, objetivo, idade em que os participantes receberam o IC, ano escolar e conclusões sobre o desempenho de leitura e escrita das crianças com IC. A síntese dos estudos foi avaliada de forma descritiva e apresentada nos Quadros 1 e 2.

\section{RESULTADOS}

O presente estudo dispõe de uma amostra inicial de 990 artigos científicos encontrados nas bases de dados BVS e PubMed $($ Medline $)=947(95,65 \%)$, LILACS $=22(2,22 \%) \mathrm{e}$ $\mathrm{SciELO}=21(2,13 \%)$. A partir deste quantitativo geral, foram subtraídos os estudos que, por sua vez, não atendiam aos critérios de inclusão e exclusão previamente estabelecidos. Apenas oito artigos foram selecionados e incluídos nesta revisão. O fluxograma abaixo (Figura 1) apresenta um resumo sobre todo o processo de busca e seleção dos artigos da pesquisa.

O Quadro 1 apresenta uma síntese dos artigos inclusos neste estudo, considerando às seguintes variáveis: autor e ano, tipo de pesquisa, revista, fator de impacto, país, amostra e objetivo.

A partir desta síntese, pode-se perceber que os países do continente europeu (Espanha) e asiático (Irã) publicaram com maior incidência estudos nesta área de interesse. Considerando o delineamento metodológico, foi possível observar no Quadro 1,

Quadro 1. Resumo dos estudos incluídos

\begin{tabular}{|c|c|c|c|c|c|c|}
\hline Autor & $\begin{array}{c}\text { Tipo de } \\
\text { pesquisa }\end{array}$ & Revista & $\begin{array}{l}\text { Fator de } \\
\text { impacto }\end{array}$ & País & Amostra & Objetivo \\
\hline Wu et al. ${ }^{(16)}$ & Transversal & $\begin{array}{c}\text { Biomed Research } \\
\text { International }\end{array}$ & 2.197 & Taiwan & $\begin{array}{l}20 \text { meninos, } 25 \text { meninas } \\
\text { entre } 8 \text { e } 13 \text { anos, usuários } \\
\text { de implante coclear. }\end{array}$ & $\begin{array}{l}\text { Examinar a escrita } \\
\text { narrativa em crianças com } \\
\text { IC e analisar os fatores } \\
\text { associados a resultados } \\
\text { desfavoráveis. }\end{array}$ \\
\hline Von Mentzer et al. ${ }^{(17)}$ & $\begin{array}{c}\text { Transversal; } \\
\text { grupo controle }\end{array}$ & $\begin{array}{l}\text { Clin Linguist } \\
\text { Phon }\end{array}$ & 1.083 & Suécia & $\begin{array}{l}22 \text { crianças, } 11 \text { com IC e } \\
11 \text { com audição normal, } \\
\text { pareadas pela idade, que } \\
\text { variou de } 5 \text { a } 8 \text { anos. }\end{array}$ & $\begin{array}{l}\text { Explorar a repetição de } \\
\text { pseudopalavras (NWR) e a } \\
\text { decodificação sem palavras } \\
\text { em crianças com audição } \\
\text { normal (AN) e em crianças } \\
\text { com IC }\end{array}$ \\
\hline Apel e Masterson ${ }^{(18)}$ & Transversal & $\begin{array}{c}\text { J Deaf Stud Deaf } \\
\text { Educ }\end{array}$ & 1.556 & EUA & $\begin{array}{l}18 \text { alunos: } 09 \text { com IC e } \\
09 \text { com audição normal, que } \\
\text { foram pareados pela idade } \\
\text { de leitura. A média de idade } \\
\text { variou de } 8 \text { a } 11 \text { anos. }\end{array}$ & $\begin{array}{l}\text { Verificar a diferença entre } \\
\text { as habilidades de ortografia } \\
\text { e, especificamente, nas } \\
\text { habilidades de consciência } \\
\text { linguística de alunos com e } \\
\text { sem (PA). }\end{array}$ \\
\hline Gallego et al.(19) & $\begin{array}{c}\text { Transversal; } \\
\text { grupo controle }\end{array}$ & Res. Dev. Disabil. & 1.872 & Espanha & $\begin{array}{l}19 \text { crianças que receberam } \\
\text { IC antes dos } 24 \text { meses de } \\
\text { idade (IC precoce) e } 19 \text { que } \\
\text { receberam após } 24 \text { meses } \\
\text { (IC tardio) com um grupo } \\
\text { controle de } 19 \text { crianças com } \\
\text { audição normal. A idade das } \\
\text { crianças foi de } 8 \text { a } 12 \text { anos. }\end{array}$ & $\begin{array}{l}\text { Comparar a compreensão } \\
\text { de leitura de sentenças } \\
\text { em crianças IC precoce e } \\
19 \text { IC tardio com um grupo } \\
\text { controle de } 19 \text { crianças com } \\
\text { audição normal. }\end{array}$ \\
\hline Domínguez et al. ${ }^{(20)}$ & Transversal & $\begin{array}{c}\text { J Deaf Stud Deaf } \\
\text { Educ. }\end{array}$ & 1.556 & Espanha & $\begin{array}{l}77 \text { crianças com PA } \\
\text { pré-lingual e IC tinham entre } \\
6 \text { e } 18 \text { anos. } 59 \text { crianças } \\
\text { PA usuárias de AASI } \\
\text { convencionais e tinham } \\
\text { entre } 8 \text { e } 17 \text { anos de idade. }\end{array}$ & $\begin{array}{l}\text { Examinar os mecanismos } \\
\text { usados por crianças surdas } \\
\text { com e sem IC para ler frases } \\
\text { e as bases linguísticas } \\
\text { (vocabulário e sintaxe) } \\
\text { subjacentes a esses } \\
\text { mecanismos de leitura }\end{array}$ \\
\hline Rezaei et al. ${ }^{(21)}$ & $\begin{array}{l}\text { Transversal; } \\
\text { grupo controle }\end{array}$ & $\begin{array}{c}\text { Int J Pediatr } \\
\text { Otorhinolaryngol. }\end{array}$ & 1.225 & Irã & $\begin{array}{l}72 \text { crianças divididas em } \\
\text { três grupos: } 24 \text { com IC, } \\
24 \text { com AASI e } 24 \text { com } \\
\text { audição normal (AN). A faixa } \\
\text { etária foi entre } 8 \text { e } 12 \text { anos. }\end{array}$ & $\begin{array}{l}\text { Examinar as habilidades } \\
\text { de leitura de crianças } \\
\text { surdas persas com IC e } \\
\text { AASI e compará-las com } \\
\text { as pessoas com audição } \\
\text { normal. }\end{array}$ \\
\hline
\end{tabular}

Legenda: PA = perda auditiva; IC = implante coclear; AASI = aparelho de amplificação sonora individual 
Quadro 1. Continuação...

\begin{tabular}{|c|c|c|c|c|c|c|}
\hline Autor & $\begin{array}{l}\text { Tipo de } \\
\text { pesquisa }\end{array}$ & Revista & $\begin{array}{l}\text { Fator de } \\
\text { impacto }\end{array}$ & País & Amostra & Objetivo \\
\hline Göçmenler e Çiprut(22) & $\begin{array}{c}\text { Transversal; } \\
\text { grupo controle }\end{array}$ & $\begin{array}{c}\text { Int J Pediatr } \\
\text { Otorhinolaryngol. }\end{array}$ & 1.225 & Turquia & $\begin{array}{l}19 \text { estudantes com IC e } \\
20 \text { alunos com audição } \\
\text { normal. A idade dos sujeitos } \\
\text { variou entre } 12 \text { e } 14 \text { anos }\end{array}$ & $\begin{array}{l}\text { Avaliar as habilidades } \\
\text { de preenchimento de } \\
\text { lacunas e erros de leitura } \\
\text { de estudantes com IC e } \\
\text { comparar seus resultados } \\
\text { com os de seus pares com } \\
\text { audição normal. Os efeitos } \\
\text { da idade de implantação e } \\
\text { do tempo total de uso do IC } \\
\text { foram analisados em relação } \\
\text { ao desenvolvimento das } \\
\text { habilidades de leitura dos } \\
\text { sujeitos. }\end{array}$ \\
\hline Pooresmaeil et al..$^{(5)}$ & $\begin{array}{l}\text { Estudo } \\
\text { descritivo } \\
\text { analítico e } \\
\text { transversal }\end{array}$ & $\begin{array}{l}\text { International } \\
\text { Journal of } \\
\text { Pediatric } \\
\text { Otorhinolaryngology }\end{array}$ & 1.225 & Irã & $\begin{array}{l}15 \text { crianças com IC e } \\
15 \text { crianças ouvintes }\end{array}$ & $\begin{array}{l}\text { Determinar a relação entre } \\
\text { compreensão de sintaxe } \\
\text { e compreensão de leitura } \\
\text { em crianças com IC e } \\
\text { ouvintes do } 3^{\circ} \text { ao } 5^{\circ} \text { ano } \\
\text { do ensino fundamental e } \\
\text { identificar a relação entre a } \\
\text { compreensão de leitura e } \\
\text { a idade de receber IC, bem } \\
\text { como também a duração do } \\
\text { tratamento fonoaudiológico } \\
\text { em crianças com IC }\end{array}$ \\
\hline
\end{tabular}

Legenda: $\mathrm{PA}$ = perda auditiva; IC = implante coclear; $\mathrm{AASI}=$ aparelho de amplificação sonora individual

Quadro 2. Caracterização dos estudos quanto aos anos escolares, idade do implante coclear, testes de avaliação e conclusões dos estudos.

\begin{tabular}{|c|c|c|c|c|c|}
\hline Autor & Amostra & $\begin{array}{l}\text { Idade do } \\
\text { implante }\end{array}$ & Ano escolar & Teste de avaliação & Conclusões \\
\hline Von Mentzer et al. ${ }^{(17)}$ & $\begin{array}{l}11 \text { crianças } \\
\text { com IC }\end{array}$ & $\begin{array}{l}\text { Média de } \\
20 \text { meses }\end{array}$ & $\begin{array}{l}\text { Escola regular; escola } \\
\text { bilíngue (língua de } \\
\text { sinais e comunicação } \\
\text { oral). }\end{array}$ & $\begin{array}{l}\text { Todas as crianças participaram } \\
\text { de um estudo de intervenção } \\
\text { em leitura assistida por } \\
\text { computador, com abordagem } \\
\text { fonética com três pontos de } \\
\text { teste (linha de base, pré- } \\
\text { intervenção e pós-intervenção); } \\
\text { Sound Information Processing } \\
\text { System (SIPS); The Test of } \\
\text { Word Reading Efficiency. }\end{array}$ & $\begin{array}{l}\text { A ausência de associações } \\
\text { significativas frequentes } \\
\text { entre repetição sem palavra } \\
\text { e decodificação sem palavras } \\
\text { em crianças com IC em } \\
\text { compaçãa com crianças } \\
\text { com audição normal sugere } \\
\text { que essas crianças usam } \\
\text { parcialmente outras estratégias } \\
\text { de decodificação para } \\
\text { compensar conhecimentos } \\
\text { fonológicos menos precisos, } \\
\text { por exemplo, lexicalizações } \\
\text { na decodificação sem } \\
\text { palavras, especificamente, } \\
\text { transformando uma palavra } \\
\text { real em uma não-palavra. } \\
\end{array}$ \\
\hline Apel e Masterson ${ }^{(18)}$ & $\begin{array}{l}9 \text { crianças } \\
\text { com IC }\end{array}$ & $\begin{array}{l}\text { Antes dos } \\
3 \text { anos }\end{array}$ & $\begin{array}{l}\text { Escola regular; escola } \\
\text { especial e regular }\end{array}$ & $\begin{array}{l}\text { TOWRE (subtestes SWE e } \\
\text { Phonetic Decoding Efficiency } \\
\text { (PDE); Teste de eficiência } \\
\text { e compreensão em leitura } \\
\text { silenciosa (TOSREC) }\end{array}$ & $\begin{array}{l}\text { As correlações entre medidas } \\
\text { de ortografia e leitura de } \\
\text { palavras reais e compreensão } \\
\text { de leitura foram menores para } \\
\text { os alunos com IC. }\end{array}$ \\
\hline
\end{tabular}

Legenda: IC = implante coclear; Teste PROLEC-R = Prova de avaliação do processo de leitura; teste READ = teste de habilidades de leitura; SMT= teste de detecção de estratégias semânticas 
Quadro 2. Continuação...

\begin{tabular}{|c|c|c|c|c|c|}
\hline Autor & Amostra & $\begin{array}{l}\text { Idade do } \\
\text { implante }\end{array}$ & Ano escolar & Teste de avaliação & Conclusões \\
\hline Gallego et al..$^{(19)}$ & $\begin{array}{l}38 \text { crianças } \\
\text { com IC }\end{array}$ & $\begin{array}{l}19 \text { antes dos } \\
24 \text { meses; } \\
\text { outras } 19 \text { entre } \\
24 \text { meses a } \\
5 \text { anos }\end{array}$ & Escola primária & $\begin{array}{l}\text { Semantic strategies detection } \\
\text { test created; WISC-IV } \\
\text { Perceptual Reasoning Index; } \\
\text { WISC-IV Forward Digit Span } \\
\text { (FDS); Peabody Picture } \\
\text { Vocabulary test (versão } \\
\text { espanhola); Sub testes } \\
\text { Estruturas gramaticais (GS) e } \\
\text { Repetição de Pseudopalavras } \\
\text { (NWR) do teste PROLEC-R }\end{array}$ & $\begin{array}{l}\text { Esses achados são discutidos } \\
\text { em relação às diferenças } \\
\text { no vocabulário receptivo e } \\
\text { na memória de curto prazo } \\
\text { e suas implicações para a } \\
\text { compreensão da leitura de } \\
\text { frases }\end{array}$ \\
\hline Domínguez et al. ${ }^{(20)}$ & $\begin{array}{l}77 \text { crianças } \\
\text { com IC }\end{array}$ & $\begin{array}{c}\text { Entre } 8 \text { meses } \\
\text { de vida e } 11 \\
\text { anos de idade }\end{array}$ & $\begin{array}{l}\text { Escola primária: } \\
1^{\circ} \text { a } 6^{\circ} \text { ano }\end{array}$ & Teste READ; Teste SMT & $\begin{array}{l}\text { Os níveis linguísticos e de leitura } \\
\text { alcançados por crianças IC } \\
\text { dependem de sua capacidade } \\
\text { de extrair informações contidas } \\
\text { no idioma falado para o qual } \\
\text { eles foram expostos. O nível } \\
\text { de leitura deste grupo não é } \\
\text { significativamente diferente do } \\
\text { nível das crianças ouvintes da } \\
\text { mesma idade. Todos os grupos de } \\
\text { participantes usaram a Estratégia } \\
\text { de Palavras- Chave. Crianças } \\
\text { surdas, incluindo aqueles que } \\
\text { usam ICs desde certa idade } \\
\text { também mostraram deficiências } \\
\text { ao lidar com palavras de conteúdo } \\
\text { quando as tarefas exigiam } \\
\text { associações profundas entre eles. }\end{array}$ \\
\hline Rezaei et al. & $\begin{array}{l}24 \text { crianças } \\
\text { com IC }\end{array}$ & $\begin{array}{l}2 \text { anos de idade } \\
\text { (em média) }\end{array}$ & $\begin{array}{l}\text { Escola básica } \\
\text { (de } 4 \text { a } 11 \text { anos), não } \\
\text { especificando o ano }\end{array}$ & $\begin{array}{l}\text { Teste Nama: Repetição } \\
\text { de pseudopalavras; leitura } \\
\text { de palavras; leitura de } \\
\text { pseudopalavras; compreensão } \\
\text { de palavras; compreensão de } \\
\text { texto. }\end{array}$ & $\begin{array}{l}\text { Considerando os achados, o } \\
\text { IC não é significativamente } \\
\text { mais eficaz que AASI para } \\
\text { melhorar as habilidades de } \\
\text { leitura. É claro que, mesmo } \\
\text { com avanços consideráveis } \\
\text { na tecnologia de AASI, muitas } \\
\text { crianças surdas } \\
\text { continuam achando a } \\
\text { alfabetização um desafio }\end{array}$ \\
\hline Pooresmaeil et al..$^{(5)}$ & $\begin{array}{l}15 \text { crianças } \\
\text { com IC }\end{array}$ & $\begin{array}{l}\text { Entre } 2 \text { e } 6 \\
\quad \text { anos }\end{array}$ & $3^{\circ}, 4^{\circ}$ e $5^{\circ}$ ano & $\begin{array}{l}\text { Reading and dyslexia test } \\
\text { (NAMA) }\end{array}$ & $\begin{array}{l}\text { Com base nos achados do } \\
\text { presente estudo, pode-vse } \\
\text { concluir que o foco na } \\
\text { compreensão da sintaxe } \\
\text { para intervenção pode } \\
\text { melhorar a compreensão } \\
\text { da leitura. Em particular, } \\
\text { parece que trabalhar em } \\
\text { estruturas complicadas } \\
\text { para as crianças ajuda a } \\
\text { melhorar sua compreensão } \\
\text { de leitura. As habilidades de } \\
\text { compreensão de sintaxe afetam } \\
\text { a compreensão de leitura em } \\
\text { crianças com IC, e a maior } \\
\text { relação pode ser encontrada } \\
\text { nas estruturas sintáticas } \\
\text { reversíveis e nas estruturas } \\
\text { relacionadas aos verbos. }\end{array}$ \\
\hline
\end{tabular}

Legenda: IC = implante coclear; Teste PROLEC-R = Prova de avaliação do processo de leitura; teste READ = teste de habilidades de leitura; SMT= teste de detecção de estratégias semânticas 


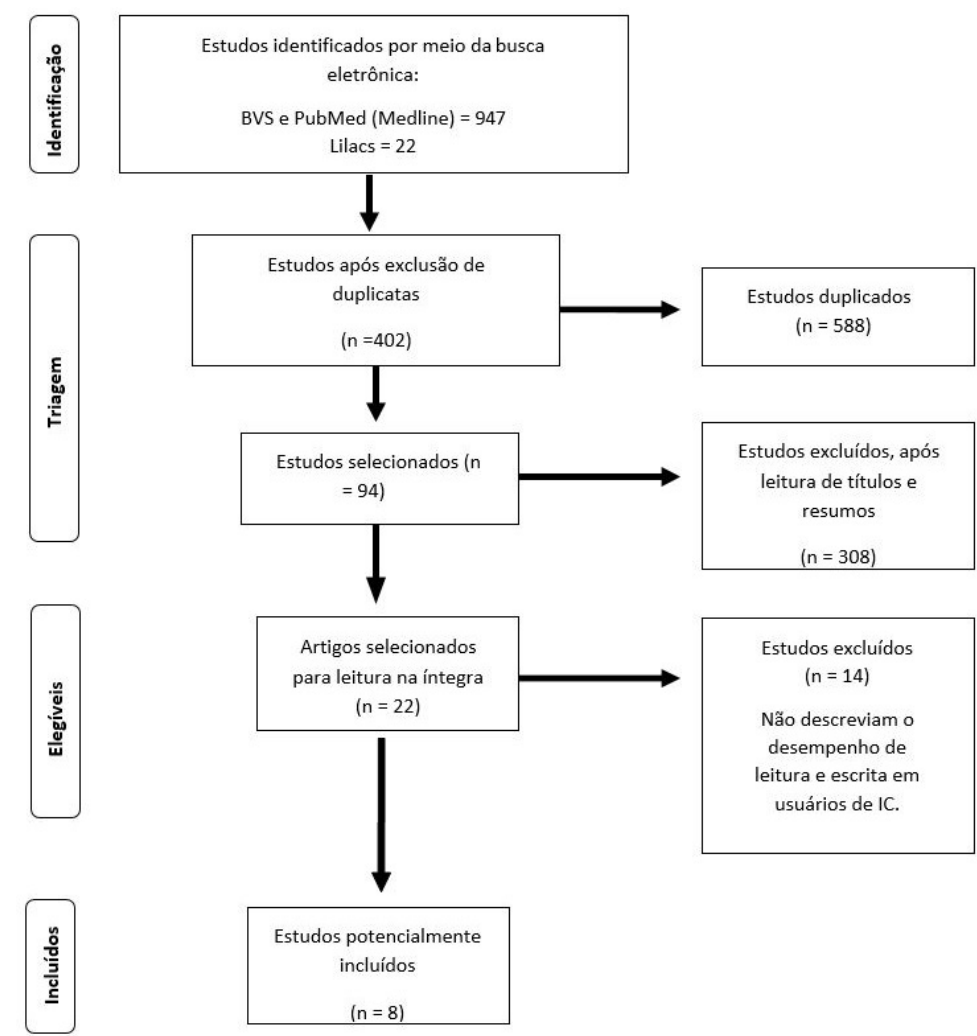

Figura 1. Fluxograma do número de artigos encontrados e selecionados após aplicação dos critérios de inclusão e exclusão Legenda: $\mathrm{IC}=$ implante coclear; $\mathrm{n}=$ número de estudos

três estudos do tipo transversal $(37,5 \%)^{(16,18,20)}$, quatro estudos do tipo transversal com grupo controle $(50 \%)^{(17,19,21,22)}$ e apenas um estudo descritivo-analítico e transversal ${ }^{(5)}$.

Durante o estudo, foram avaliados 419 escolares, sendo que $238(56,80 \%)$ são usuários de IC, que tinham como grupo controle $181(43,20 \%)$ crianças com audição normal. Com isso, verifica-se a faixa etária de 5 a 18 anos de idade.

Considerando-se os métodos avaliativos utilizados durante os estudos, foi observado que os autores não seguiram um padrão. Os testes foram escolhidos de acordo com a preferência de cada autor, referindo as habilidades avaliadas de leitura e escrita.

O Quadro 2 apresenta a categorização dos estudos quanto aos testes de avaliação utilizados nos artigos inclusos nesta revisão, além de apresentar o tamanho da amostra, o ano escolar que elas frequentavam, a média da idade em que os participantes receberam o IC e as devidas conclusões de cada estudo.

\section{DISCUSSÃo}

O presente estudo foi elaborado com o objetivo de descrever o desempenho de leitura e escrita em usuários de IC por meio de uma revisão integrativa. Diante dos resultados obtidos nesta pesquisa, verificou-se que usuários com IC possuem um atraso significativo durante o processo de desenvolvimento de leitura e de escrita, sendo este considerado ainda maior, quando comparados com indivíduos de audição normal. Vários são os fatores que podem influenciar neste desenvolvimento.

Em meio à seleção, não foi encontrado estudo brasileiro publicado em plataformas online referente a esta temática, o que demonstra a pouca produção sobre o tema no país.
Por conseguinte, houve maior incidência de publicações internacionais. Os resultados obtidos identificam os países do continente europeu e asiático como os países com maior incidência de estudos relacionados ao desempenho de leitura e escrita em usuários de IC. Provavelmente, isso acontece porque o uso do IC tem maior histórico como uma intervenção eficaz na reabilitação auditiva nesses continentes.

Quanto ao tipo de estudo, o delineamento metodológico com maior quantitativo foi o de estudos transversais. Provavelmente, porque neste tipo de estudo é possível analisar dados coletados em um único período. Sendo assim, quatro estudos, além de serem transversais, utilizaram grupo controle $(50 \%)^{(17,19,21,22)}$. Nestes, os autores necessitaram de um pareamento entre usuários de IC e ouvintes. Os métodos de avaliação utilizados pelos autores dos artigos estudados não expressaram um padrão, mas mostraram eficácia para avaliar e caracterizar o desempenho de leitura e de escrita em usuários de IC, considerando que são testes padronizados e validados na área de leitura e escrita para esses usuários. Nenhum dos estudos selecionados é brasileiro, o que dificulta aos clínicos e pesquisadores o conhecimento de testes que poderiam ser eficazes para avaliação dessas crianças no contexto brasileiro.

Seguindo este pressuposto, todos os artigos selecionados para a pesquisa afirmaram que o desempenho de leitura e de escrita em usuários de IC é considerado menor em comparação ao dos ouvintes. Outros estudos, além desta revisão ${ }^{(8,23)}$, corroboram com os resultados obtidos acima. Eles destacam que crianças com IC possuem um atraso significativo de 2 a 3 anos no desenvolvimento das habilidades de leitura e de escrita, quando comparadas a escolares de audição normal no mesmo ano escolar ${ }^{(8,23)}$. 
Apenas dois estudos ${ }^{(16,18)}$ apresentaram uma análise específica da escrita em crianças com IC. Os resultados mostram que, em comparação com crianças com audição normal, crianças com IC escrevem textos com menor quantidade de palavras, história sem ponto principal e, ao descrever imagens e objetos, não mencionam ações e sentimentos dos personagens apresentados. Autores sugerem que a ausência de capacidade de desenvolver e expressar uma história pode estar associada a um input auditivo insatisfatório ${ }^{(16)}$.

Crianças usuárias de IC oralizadas com abordagem aurioral para reabilitação auditiva devem seguir as mesmas etapas na aquisição de leitura e de escrita em relação às crianças ouvintes ${ }^{(8)}$. Entretanto, mesmo que o IC propicie sensações auditivas às crianças com perda auditiva severa/profunda, a aquisição da linguagem oral e escrita parece continuar deficitária, quando comparada aos colegas com audição normal ${ }^{(22)}$, sendo necessário um programa de reabilitação auditiva mais intensivo nos primeiros anos de vida e com objetivo maior de remediação para o atraso de consciência fonológica nos anos escolares inicias para que elas atinjam melhores níveis de compreensão das informações recebidas e melhor domínio no processo de alfabetização. Segundo os artigos estudados, a dificuldade no desempenho de leitura e escrita em usuários de IC pode estar associada a um déficit na linguagem receptiva desses usuários, ou seja, eles necessitam de maiores experiências sonoras para entender o que está sendo comunicado oralmente.

A grande dificuldade do usuário de IC ainda é desenvolver e compreender a linguagem oral, o que influencia diversas vezes no aprendizado da linguagem escrita. Estudo brasileiro, por exemplo, defende o apoio visual, a contação e a dramatização de histórias como estratégias para o ensino de leitura e escrita de crianças com IC. Além do treinamento auditivo, as narrativas também estimulam a memória auditiva sequencial, o aumento do vocabulário e os aspectos da linguagem, como a semântica e prosódia, fundamentais para o desenvolvimento da consciência fonológica.

Desta feita, é de suma importância que sejam realizadas contação de histórias por meio de um programa de gerenciamento entre leitores e mediadores, podendo ser iniciado desde bebês ${ }^{(24,25)}$. As crianças usuárias de IC devem ser também beneficiadas pelo trabalho da fonoaudiologia educacional, assim como do atendimento educacional adaptado e especializado para desenvolver habilidades de leitura e escrita ${ }^{(26)}$ dentro da proposta de inclusão de cada país.

A partir da análise das tarefas de avaliação que envolviam o desempenho de leitura e de escrita em usuários de IC e de AASI, foi possível observar um achado de apenas dois artigos $(25 \%)^{(20,21)}$. Um estudo espanhol mostrou que os níveis de leitura entre os grupos não apresentaram diferenças, pois ambos possuem dificuldades ao lidar com palavras mais complexas, principalmente, quando são exigidas associações entre elas ${ }^{(20)}$.

Já estudiosos israelenses afirmam que o uso do IC não é significativamente mais eficiente comparado ao uso do AASI para aperfeiçoar habilidades de leitura ${ }^{(21)}$. Talvez porque outras variáveis, tais como o processo de inclusão e o status sociocultural dos pais, precisam ser consideradas e não apenas o tipo de dispositivo eletrônico auditivo utilizado. Com isso, pode-se afirmar que pais com estilo de frases mais complexas poderão influenciar diretamente na compreensão e na formulação das narrativas dessas crianças com IC. Os usuários, ao serem expostos a este estilo de frases mais complexas, possuem experiências mais elaboradas em relação ao contexto que está incluído.

Entretanto, um estudo confronta o resultado das pesquisas citadas anteriormente, afirmando que a compreensão da leitura entre as crianças usuárias de IC é significativamente melhor do que a de crianças usuárias de $\mathrm{AASI}^{(27)}$. Os achados nos aspectos de percepção auditiva são inquestionáveis quanto ao melhor benefício proporcionado pelo IC em comparação ao AASI para usuários com perda auditiva severa/profunda pré-lingual. Contudo, tal desempenho, de ambos os grupos, ainda se encontra aquém quando comparado ao de crianças ouvintes. Novamente, é importante considerar outras particularidades necessárias na fundamentação desses achados, como as estratégias utilizadas por esses grupos para identificação de um conteúdo em um texto.

A dificuldade na compreensão da leitura desses indivíduos pode estar associada a um déficit no processamento sintático das frases, ou seja, os indivíduos com perda auditiva severa/profunda não recebem na íntegra as regras gramaticais que determinam a estrutura de uma sentença corretamente, fazendo com que, possivelmente, a escrita torne-se incoerente em consideração à ideia principal do texto. Defendendo esse pressuposto, segundo estudos, indivíduos com perda auditiva realizam estratégias semânticas que envolvem a compreensão de sentenças ${ }^{(28)}$. Essa estratégia busca identificar o conteúdo da frase, selecionando palavras-alvo como fechamento de ideias, ignorando qualquer outra informação apresentada.

$\mathrm{Na}$ avaliação das relações que envolviam o desenvolvimento das habilidades de leitura e de escrita, considerando as variáveis "idade de implantação" e o "tempo total de uso do IC", foi possível observar apenas três artigos $(37,5 \%)^{(5,19,22)}$. Tais resultados demonstram que os indivíduos implantados precocemente e/ou tardiamente possuem diferenças significativas quanto ao desempenho de leitura e de escrita em comparação aos que possuem audição normal ${ }^{(5,19,22)}$.

Crianças implantadas precocemente usam preferencialmente pistas sintáticas ao tentar compreender e completar frases lidas, significando que, na ausência de um entendimento adequado, essas crianças buscam palavras que se inserem morfossintaticamente na frase, o que se assemelha com habilidades de crianças que possuem audição normal ${ }^{(19)}$. Para as crianças implantadas tardiamente, faz-se o uso de pistas semânticas para esta compreensão, conforme o uso da estratégia de palavras-chave. Porém, em alguns momentos, essas crianças empregam sinais sintáticos, usando as duas estratégias ${ }^{(19)}$.

O único estudo descritivo, analítico e transversal incluso nesta revisão afirma que mesmo que a estratégia de compreensão das crianças implantadas precocemente se assemelhe com as crianças ouvintes, pode haver uma diferença no desempenho de leitura entre os grupos ${ }^{(22)}$. Neste sentido, pesquisas demonstram que crianças com perda auditiva profunda que recebem o IC precocemente têm desempenho significativamente melhor em relação às que recebem o implante tardiamente ${ }^{(29,30)}$.

Apesar dos benefícios auditivos que o IC pode oferecer a uma criança com perda auditiva, a capacidade de organizar uma história por escrito ou expressá-la por meio da leitura poderá ser aperfeiçoada com o aumento das experiências sonoras desses usuários. Deste modo, quanto mais exposição ao input auditivo essa criança tiver, melhor será sua capacidade de codificar e decodificar as palavras. Sendo assim, verifica-se a importância dos programas de reabilitação auditiva após IC, além de um atendimento educacional adequado aos usuários de IC em seu ambiente escolar.

\section{CONCLUSÃO}

Esta revisão de literatura indicou que, mesmo com o uso do IC, o desempenho de leitura e de escrita está aquém do esperado para faixa etária e ano escolar. Além disso, os usuários 
de IC apresentaram desempenho inferior aos dos ouvintes quando comparados a estes. Esse déficit pode estar associado à linguagem receptiva desses usuários, ou seja, eles precisam de maiores experiências sonoras para compreender o que estão ouvindo, para, a partir disso, aperfeiçoar suas habilidades de codificação (leitura) e decodificação (escrita).

Além disso, implementações no processo de inclusão podem favorecer melhores resultados no desempenho escolar de usuários de IC, como um programa de gerenciamento de leitura sugerido por pesquisadores. A partir dos estudos analisados, percebe-se escassez de pesquisas sobre o desempenho de leitura e escrita em usuários de IC no Brasil, necessitando, portanto, de mais estudos e publicações referentes a esta temática.

\section{REFERÊNCIAS}

1. Neves AJ, Verdu ACMA, Mortarimoret AL, Silva LTN. As implicações do implante coclear para desenvolvimento das habilidades de linguagem: uma revisão da literatura. Rev CEFAC. 2015;17(5):1643-56. http:// dx.doi.org/10.1590/1982-021620151755315.

2. Sobreira ACO, Capo BM, Santos TS, Gil D. Desenvolvimento de fala e linguagem na deficiência auditiva: relato de dois casos. Rev CEFAC. 2015;17(1):308-17. http://dx.doi.org/10.1590/1982-021620152314.

3. Sharma A, Dorman MF, Kral A. The infuence of a sensitive period on central auditory development in children with unilateral and bilateral cochlear implants. Hear Res. 2005;203(1-2):134-43. http://dx.doi. org/10.1016/j.heares.2004.12.010. PMid:15855038.

4. Moretti CAM, Ribas A, Guarinello AC, Rosa MRD. Hearing and language development scale in cochlear implanted children. Audiol Commun Res. 2018;23:e1895.

5. Pooresmaeil E, Mohamadi R, Ghorbani A, Kamali M. The relationship between comprehension of syntax and reading comprehension in cochlear implanted and hearing children. Int J Pediatr Otorhinolaryngol. 2019;121:114-9. http://dx.doi.org/10.1016/j.ijporl.2019.03.004. PMid:30878557.

6. Djourno A, Eyries $\mathrm{C}$. Prothèse auditive par excitation électrique a distance du nerf sensoriel a l' aide d' un bobinage inclus a demeure. Presse Med. 1957;63(65):14-7.

7. Tanamati LF, Bevilacqua MC, Costa AO. Cochlear implant in postlingual children: functional results 10 years after the surgery. Rev Bras Otorrinolaringol. 2012;78(2):103-10. PMid:22499377.

8. Pinheiro ABSM, Yamada MO, Bevilacqua MC, Crenitte PAP. Assessing skills of school children with cochlear implant. Rev CEFAC. 2012;14(5):826-35. http://dx.doi.org/10.1590/S1516-18462012005000059.

9. Capovilla FC. Cochlear implant as a tool for the deaf child's language development. Rev. Bras. Cresc. Des. Hum. 1998;8(1-2):76-84.

10. Santos SLR. Caracterização de desempenhos envolvidos na leitura e na escrita em crianças com deficiência auditiva [dissertação]. Bauru: Universidade Estadual Paulista; 2012.

11. Brazorotto JS. Crianças usuárias de implante coclear: desempenho acadêmico, expectativa dos pais e professores [tese]. São Carlos: Universidade Federal de São Carlos; 2009.

12. Salesa Batlle E. Avances principales de la audiología actual: previsión de futuro: major advances in current audiology. Rev Logop Fon Audiol. 2012;32(4):149-51. http://dx.doi.org/10.1016/j.rlfa.2012.10.002.

13. Duarte JL, Brazorotto JS. Analyses of strategies used in a pedagogic therapeutic group to enhance the development of writing in hearing impaired children. Rev Bras Educ Espec. 2009;15(3):471-84. http:// dx.doi.org/10.1590/S1413-65382009000300010.
14. Dias N, Seabra AG. Funções executivas: desenvolvimento e intervenção Temas desenvolv. 2013;19(107):206-12.

15. Souza MT, Silva MD, Carvalho R. Integrative review: what is it? How to do it? Einstein. 2010;8(1):102-6. http://dx.doi.org/10.1590/ s1679-45082010rw1134. PMid:26761761.

16. Wu CM, Ko HC, Chen YA, Tsou YT, Chao WC. Written language ability in mandarin-speaking children with cochlear implants. BioMed Res Int. 2015;2015:282164. http://dx.doi.org/10.1155/2015/282164. PMid:26236722.

17. Von Mentzer CN, Lyxell B, Shalén B, Dahlström O, Lindgren M, Ors M, et al. Segmental and suprasegmental properties in nonword repetition? An explorative study of the associations with nonword decoding in children with normal hearing and children with bilateral cochlear implants. Clin Linguist Phon. 2014;29(3):216-35. http:// dx.doi.org/10.3109/02699206.2014.987926. PMid:25489675.

18. Apel K, Masterson JJ. Comparing the spelling and reading abilities of students with cochlear implants and students with typical hearing. J Deaf Stud Deaf Educ. 2015;20(2):125-35. http://dx.doi.org/10.1093/ deafed/env002. PMid:25693579.

19. Gallego C, Martín-Aragoneses MT, López-Higes R, Pisón G. Semantic and syntactic reading comprehension strategies used by deaf children with early and late cochlear implantation. Res Dev Disabil. 2016;4950:153-70. http://dx.doi.org/10.1016/j.ridd.2015.11.020. PMid:26704778.

20. Domínguez AB, Carrilo MS, González VG, Alegria J. How do deaf children with and without cochlear implants manage to read sentences: the key word strategy. J Deaf Stud Deaf Educ. 2016;21(3):280-92. http://dx.doi.org/10.1093/deafed/enw026. PMid:27151899.

21. Rezaei M, Rashedi V, Morasae EK. Reading skills in Persian deaf children with cochlear implants and hearing aids. Int J Pediatr Otorhinolaryngol. 2016;89:1-5. http://dx.doi.org/10.1016/j. ijporl.2016.07.010. PMid:27619019.

22. Göçmenler H, Çiprut A. Evaluation of gap filling skills and reading mistakes of cochlear implanted and normally hearing students. Int $\mathbf{J}$ Pediatr Otorhinolaryngol. 2018;109:27-30. http://dx.doi.org/10.1016/j. ijporl.2018.03.014. PMid:29728179.

23. Spencer LJ, Barker BA, Tomblin JB. Exploring the language and literacy outcomes of pediatric cochlear implant users. Ear Hear. 2003;24(3):23647. http://dx.doi.org/10.1097/01.AUD.0000069231.72244.94. PMid:12799546.

24. Oliveira JP, Bonkill E, Braga TMS 3rd, Schier AC. Produção de conhecimento sobre narrativas orais: contribuições para as investigações em linguagem infantil. Rev CEFAC. 2013;15(1):207-14. http://dx.doi. org/10.1590/S1516-18462012005000108.

25. Evans D. Estilos cognitivo-afetivos e construção da linguagem através das narrativas. Constr Psicopedag. 2013;21(22):67-85.

26. Barbosa RS. Intervenção pedagógica para ensino de leitura, escrita e aritmética para uma criança com implante coclear [tese]. São Carlos: Universidade Federal de São Carlos; 2015.

27. Marschark M, Rhoten C, Fabich M. Effects of cochlear implants on children's reading and academic achievement. J Deaf Stud Deaf Educ. 2007;12(3):269-82. http://dx.doi.org/10.1093/deafed/enm013. PMid: 17526867.

28. Domínguez AB, Pérez I, Alegria J. Reading in deaf students: the role of the cochlear implant. Infanc Aprendiz. 2012;35(3):327-41.

29. Nicholas JG, Geers AE. Effects of early auditory experience on the spoken language of deaf children at 3 years of age. Ear Hear. 2006;27(3):286-98. http://dx.doi.org/10.1097/01.aud.0000215973.76912. c6. PMid:16672797.

30. Holt RF, Svirsky MA. An exploratory look at pediatric cochlear implantation: is earliest always best? Ear Hear. 2008;29(4):492-511. http://dx.doi.org/10.1097/AUD.0b013e31816c409f. PMid:18382374. 